\title{
Effect of self-reported walking difficulty on bone mass and bone resorption marker in Japanese people aged 40 years and over
}

\author{
Yasuyo Abe ${ }^{1 *}$, Takayuki Nishimura', Kazuhiko Arima1', Mitsuo Kanagae ${ }^{1,2}$, Satoshi Mizukami ${ }^{1,2}$, Yoshihito Tomita ${ }^{1,2}$,
} Takuhiro Okabe ${ }^{1,2}$, Hisashi Goto ${ }^{3}$, Itsuko Horiguchi ${ }^{4}$ and Kiyoshi Aoyagi ${ }^{1}$

\begin{abstract}
Background: This study aimed to examine the association of walking difficulty with bone mass or bone turnover among community-dwelling Japanese people aged 40 years and older.

Methods: We studied 1097 community-dwelling Japanese people aged 40 years and older (379 men and 718 women) who were invited to participate in periodic health examinations in 2006-2009. Walking difficulty was defined as having difficulty walking $100 \mathrm{~m}$ on a level surface (self-administered questionnaire). Calcaneal stiffness index (bone mass) was measured by quantitative ultrasound. Spot urine samples were collected, and urinary $\mathrm{N}$-terminal cross-linking telopeptide of type I collagen (NTx) was measured. Values were corrected for creatinine (Cre) concentration.

Results: The prevalence of walking difficulty was significantly higher in women than in men (7.4 vs. $3.4 \%, p=0.011)$ and significantly increased with age in men ( $p$ for trend $=0.02$ ) and women ( $p$ for trend $<0.001)$. In univariate analysis, men and women with walking difficulty were older $(p<0.001)$ and had a lower stiffness index $(p<0.001)$, compared with those without walking difficulty. Among women, individuals with walking difficulty had significantly higher urinary $N T x /$ Cre than those without walking difficulty $(p<0.001)$; however, this was not so among men $(p=0.39)$. Multiple regression analysis adjusted for age, weight, and menopausal status showed a significant association between walking difficulty and lower stiffness index in men $(p=0.004)$ and women $(p=0.005)$. In women, walking difficulty was significantly associated with higher NTx/Cre $(p=0.001)$, but not in men $(p=0.35)$.
\end{abstract}

Conclusions: Walking difficulty may contribute to low bone mass in both sexes but might cause high bone turnover in women only.

Keywords: Walking difficulty, Bone mass, Calcaneal stiffness index, Bone turnover

\section{Background}

Low bone mass and accelerated bone turnover contribute to osteoporotic fractures. Quantitative ultrasound (QUS) parameters of the calcaneus predict fracture risk among elderly men and women [1-5]. Biochemical markers of bone turnover predict fracture risk in elderly women [6-9]. Cross-linked N-telopeptide of type I collagen (NTx) is one of the biochemical markers of bone resorption and is widely used in epidemiological $[6,7]$ and clinical studies [10].

\footnotetext{
*Correspondence: yasuyo-a@nagasaki-u.ac.jp

1 Department of Public Health, Nagasaki University Graduate School of Biomedical Sciences, Nagasaki, Japan

Full list of author information is available at the end of the article
}

Physical activity influences bone mass and bone turnover. High levels of physical activity are associated with high QUS parameters among elderly women [11-16]. Increased physical activity is effective in reducing the levels of bone resorption markers among elderly men and women [17-20].

Walking ability is an important element of physical activity. Thus far, the effect of walking difficulty on bone mass or bone turnover has not well been studied. Kitagawa et al. [21] reported the association between levels of walking activity and levels of QUS parameters or bone turnover in elderly women, but there are no data in men. Chen et al. [22] reported the association between measures of mobility (graded using a four-point 
scale as follows: walks unaided, uses stick, uses frame, needs wheelchair) and bone turnover markers among older people in residential care facilities, but results were not shown separately for men and women.

The self-reported walking difficulty assessed by asking people if they have any difficulty walking $100 \mathrm{~m}$ on a level surface [23] is considered to detect moderate immobility [24]. This simple and easy to apply question has been applied in many aging studies [23, 25, 26]. It would be necessary to examine the association of walking difficulty with bone health status in order to evaluate their physiological adaptations to moderate immobility.

The purpose of this study was to assess the association of walking difficulty (chronic immobility) with bone mass and bone turnover among community-dwelling Japanese men and women aged 40 years and older.

\section{Methods}

Subjects were community-dwelling people aged 40 years and older at Nagasaki Prefecture, Japan, who were invited to participate in periodic health examinations in 20062009. A total of 1097 people ( 379 men and 718 women) participated in the study. Subjects were asked if they had difficulty walking $100 \mathrm{~m}$ on a level surface (walking difficulty). Calcaneal stiffness index (bone mass) was measured using a Lunar Achilles device (GE Lunar Corp., Madison, WI). Spot urine samples (8:00-10:00 am) were collected. Urinary $\mathrm{N}$-terminal cross-linking telopeptide of type I collagen (NTx), a marker of bone resorption, was measured using an enzyme immunoassay. Values were corrected for creatinine (Cre) concentration. Height $(\mathrm{m})$ and weight $(\mathrm{kg})$ were measured with light clothing and without shoes, and body mass index (BMI) was calculated as weight $(\mathrm{kg}) /$ height $^{2}\left(\mathrm{~m}^{2}\right)$.

Written informed consent was obtained from all the subjects prior to the study. The ethics committee of Nagasaki University Graduate School of Biomedical Sciences approved the study.

\section{Statistical analysis}

The student $t$ test was used to determine the significance of differences between subjects with and without walking difficulty. Cochran-Armitage trend test was used to evaluate differences in the prevalence of walking difficulty among age groups (40-49, 50-59, 60-69, 70-79, and 80 and over). Because urinary NTx/Cre had a skewed distribution, logarithmic transformation was performed for the analysis. Student's $t$ test was used to examine the difference in variables between individuals with and without walking difficulty. Multiple linear regression analysis was used to explore the effects of walking difficulty on calcaneal stiffness index or urinary NTx/Cre, adjusting for age, weight, and menopausal status. Data were analyzed using the Statistical Analysis System Version 9.2. (SAS Institute Inc. Cary, NC).

\section{Results}

Table 1 shows characteristics of subjects. Mean age (SD) was 65.6 (9.8) years in men and 65.0 (9.6) years in women. The prevalence of walking difficulty was significantly higher in women than in men ( 7.4 vs. $3.4 \%, p=0.011)$.

Table 2 shows prevalence of walking difficulty by age groups. The prevalence of walking difficulty significantly increased with age both in men $(p$ for trend $=0.02)$ and in women ( $p$ for trend $<0.001$ ).

In univariate analysis, men and women with walking difficulty were significantly older ( $p=0.0008$ in men, $p<0.0001$ in women) and had a significantly lower calcaneal stiffness index ( $p=0.0006$ in men, $p<0.0001$ in women) compared with those without walking difficulty (Table 3 ). Among women, individuals with walking difficulty had significantly higher urinary NTx/Cre than those without walking difficulty $(p<0.0001)$, but no significant association was observed among men $(p=0.39)$.

Multiple linear regression analysis adjusted for age, weight, and menopausal status showed a significant association between walking difficulty and lower calcaneal stiffness index in men $(p=0.004)$ and women $(p=0.005)$ (Table 4$)$. In women, walking difficulty was significantly associated with higher $\mathrm{NTx} / \mathrm{Cre}(p=0.001)$, but not in men $(p=0.35)$.

\section{Discussion}

We showed that walking difficulty (having difficulty walking $100 \mathrm{~m}$ on a level surface) was associated with a lower calcaneal stiffness index (bone mass) in both sexes and with higher NTx/Cre (bone resorption) only in women. Bone metabolism status might differ between men and women. To the best of our knowledge, this is the first

Table 1 Characteristics of study subjects

\begin{tabular}{lll}
\hline & Men $(n=379)$ & Women $(n=718)$ \\
\hline Age (years) & Mean (SD) & Mean (SD) \\
Weight $(\mathrm{kg})$ & $65.6(9.8)$ & $65.0(9.6)$ \\
Height (cm) & $62.7(9.1)$ & $52.6(8.4)$ \\
Body mass index $\left(\mathrm{kg} / \mathrm{m}^{2}\right)$ & $162.9(6.1)$ & $150.7(6.0)$ \\
Calcaneal stiffness index & $23.6(2.9)$ & $23.1(3.4)$ \\
Urinary NTx/Cre & $86.5(17.4)$ & $69.9(12.3)$ \\
(nmol BCE/mmol·CRE) & $36.2(16.9)$ & $56.6(26.8)$ \\
& & Number (\%) \\
Walking difficulty & Number (\%) & $53(7.4)^{*}$ \\
Postmenopausal & $13(3.5)$ & $655(91.2)$ \\
\hline
\end{tabular}

NTx N-terminal cross-linking telopeptide of type I collagen, Cre creatinine, $B C E$ bone collagen equivalents

${ }^{*} p=0.011$ vs. men 
Table 2 Prevalence of walking difficulty by age group

\begin{tabular}{cccccccc}
\hline Age (years) & $40-49$ & $50-59$ & $60-69$ & $70-79$ & $\begin{array}{l}80 \text { and } \\
\text { over }\end{array}$ & $\begin{array}{l}p \text { for } \\
\text { trend }^{\mathrm{a}}\end{array}$ \\
\hline & & & & & & & \\
& Men & & & & & \\
& $(n=24)$ & $(n=70)$ & $(n=139)$ & $(n=119)$ & $(n=27)$ & \\
& $0(0.0)$ & $2(2.9)$ & $2(1.4)$ & $6(5.0)$ & $3(11.1)$ & 0.02 \\
& Women & & & & & \\
& $(n=54)$ & $(n=146)$ & $(n=274)$ & $(n=213)$ & $(n=31)$ & \\
& $0(0.0)$ & $3(2.1)$ & $18(6.6)$ & $24(11.3)$ & $8(25.8)$ & $<0.0001$
\end{tabular}

Values are number (\%)

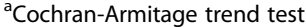

study to examine the association of walking difficulty (chronic immobility) with bone resorption markers separately for men and women.

Physical activity contributes to high QUS parameters [1-5]. Kitagawa et al. [21] showed that number of daily walking steps was positively associated with QUS parameters (speed of sound, broadband ultrasound attenuation, or stiffness index) among postmenopausal Japanese women. Sun et al. [27] showed that gait parameters were associated with calcaneal stiffness index among community-dwelling Japanese elderly women. We showed a significant association between walking difficulty and lower calcaneal stiffness index in both sexes, suggesting that chronic moderate immobility may influence low bone mass in men and women.

Chronic immobility could markedly affect bone metabolism in elderly people [28]. Sorva et al. [28] found that serum carboxyterminal cross-linked telepeptide of type I

Table 3 Comparison of variables between subjects with and without walking difficulty

\begin{tabular}{|c|c|c|c|}
\hline & With & Without & $p^{\mathrm{a}}$ \\
\hline & Men & & \\
\hline & $(n=13)$ & $(n=366)$ & \\
\hline Age (years) & $72.6(9.0)$ & $65.4(9.7)$ & 0.0008 \\
\hline Weight (kg) & $62.0(9.6)$ & $62.7(9.1)$ & 0.78 \\
\hline Body mass index $\left(\mathrm{kg} / \mathrm{m}^{2}\right)$ & $24.2(3.6)$ & $23.6(2.9)$ & 0.41 \\
\hline Calcaneal stiffness index & $70.2(13.4)$ & $87.1(17.3)$ & 0.0006 \\
\hline \multirow[t]{3}{*}{$\log (\mathrm{NTx} / \mathrm{Cre})(\mathrm{nmol} \mathrm{BCE} / \mathrm{mmol} \cdot \mathrm{CRE})$} & $3.6(0.53)$ & $3.5(0.43)$ & 0.39 \\
\hline & Women & & \\
\hline & $(n=53)$ & $(n=665)$ & \\
\hline Age (years) & $72.1(8.1)$ & $64.4(9.5)$ & $<0.0001$ \\
\hline Weight & $51.9(8.9)$ & $52.7(8.4)$ & 0.53 \\
\hline Body mass index $\left(\mathrm{kg} / \mathrm{m}^{2}\right)$ & $24.0(3.5)$ & $23.1(3.4)$ & 0.07 \\
\hline Calcaneal stiffness index & $60.0(13.1)$ & $70.7(14.1)$ & $<0.0001$ \\
\hline Log (NTx/Cre) (nmol BCE/mmol·CRE) & $4.2(0.48)$ & $3.9(0.48)$ & $<0.0001$ \\
\hline
\end{tabular}

NTX N-terminal cross-linking telopeptide of type I collagen, Cre creatinine, $B C E$ bone collagen equivalents

${ }^{\mathrm{a}} t$ test
Table 4 Association of walking difficulty to calcaneal stiffness index or urinary NTx/Cre: results of multiple regression analysis adjusting for age, weight, and menopausal status

\begin{tabular}{llll}
\hline Outcome & Estimate & Standard error & $p$ \\
\hline Men & & & \\
Calcaneal stiffness index & -13.7 & 4.7 & 0.004 \\
Log (NTx/Cre) & 0.11 & 0.12 & 0.35 \\
Women & & & 0.005 \\
Calcaneal stiffness index & -4.7 & 1.7 & 0.001 \\
Log (NTx/Cre) & 0.22 & 0.07 &
\end{tabular}

collagen, a bone resorption marker, was elevated in bedridden elderly subjects. Bischoff et al. [29] found that among residents of a long-stay geriatric ward, more immobile subjects had significantly higher excretion rates for urinary deoxypyridinoline, a bone resorption marker. On the other hand, Chen et al. [22] found that in elderly men and women living in residential aged care facilities, ageand gender-adjusted levels of serum carboxyterminal telopeptide of type I collagen, a bone resorption marker, were significantly higher in men and women with poorer mobility, but no significant association was found after further adjustment for weight, parathyroid hormone, and other covariates. Thus, the relationship between chronic immobility and bone resorption markers is controversial. Furthermore, few studies have assessed men separately, and the effect of relatively moderate immobility on bone resorption markers has not yet been determined. Further study is needed to elucidate the effect of moderate immobility on bone resorption markers, considering possible differences between men and women.

This study has several limitations. First, because this study was conducted in a cross-sectional setting, these results do not show a causal relationship. Longitudinal studies are required to confirm whether changes in QUS parameters and NTx levels are affected by walking difficulty. Second, study participants were mobile enough to attend the examination site, contributing to selection bias. Third, potential confounders that can affect immobility and increased bone resorption, such as lifestyle factors (smoking, alcohol consumption, and exercise), vitamin D deficiency, low calcium intake, secondary hyperparathyroidism, current medications, years after menopause, and renal insufficiency [22], were not available in this study.

In conclusion, walking difficulty may contribute to low bone mass in both sexes but might cause high bone turnover only in women. Our findings suggest that chronic immobility is an important factor contributing to the low bone mass and high bone turnover especially in women. Studies of bone mass and biochemical markers of bone turnover in various at-risk populations should also consider the influence of mobility. 


\section{Abbreviations}

BCE: Bone collagen equivalents; BMl: Body mass index; Cre: Creatinine; NTx: Urinary cross-linked N-telopeptide of type I collagen; QUS: Quantitative ultrasound; SD: Standard deviation

\section{Authors' contributions}

YA contributed to study design and data correction, and drafted the manuscript; TN contributed to data analysis and made critical revisions to the manuscript; KA made critical revisions to the manuscript; MK made critical revisions to the manuscript; SM contributed to data collection and made critical revisions to the manuscript; YT and TO contributed to analysis and made critical revisions to the manuscript. $\mathrm{HG}$ and $\mathrm{IH}$ made critical revisions to the manuscript. KA supervised the study, contributed to analysis and interpretation of data, and made critical revisions to the manuscript. All authors read and approved the final manuscript.

\section{Competing interests}

The authors declare that they have no competing interests.

\section{Ethics approval and consent to participate}

Written informed consent was obtained from all the subjects prior to the study. The ethics committee of Nagasaki University Graduate School of Biomedical Sciences approved the study.

\section{Author details}

'Department of Public Health, Nagasaki University Graduate School of Biomedical Sciences, Nagasaki, Japan. ${ }^{2}$ Department of Rehabilitation, Nishi-Isahaya Hospital, Isahaya, Japan. ${ }^{3}$ Goto Health Care Office, Nagasaki, Japan. ${ }^{4}$ Center for Public Relations Strategy, Nagasaki University, Nagasaki, Japan.

Received: 3 April 2016 Accepted: 30 September 2016

Published online: 11 October 2016

\section{References}

1. Bauer DC, Gluer CC, Cauley JA, Vogt TM, Ensrud KE, Genant HK, et al. Broadband ultrasound attenuation predicts fractures strongly and independently of densitometry in older women. A prospective study. Study of Osteoporotic Fractures Research Group. Arch Intern Med. 1997;157(6):629-34.

2. Fujiwara S, Sone T, Yamazaki K, Yoshimura N, Nakatsuka K, Masunari N, et al. Heel bone ultrasound predicts non-spine fracture in Japanese men and women. Osteoporos Int. 2005;16(12):2107-12.

3. Hans D, Dargent-Molina P, Schott AM, Sebert JL, Cormier C, Kotzki PO, et al. Ultrasonographic heel measurements to predict hip fracture in elderly women: the EPIDOS prospective study. Lancet. 1996:348(9026):511-4.

4. Huopio J, Kroger H, Honkanen R, Jurvelin J, Saarikoski S, Alhava E. Calcaneal ultrasound predicts early postmenopausal fractures as well as axial BMD. A prospective study of 422 women. Osteoporos Int. 2004;15(3):190-5.

5. Miller PD, Siris ES, Barrett-Connor E, Faulkner KG, Wehren LE, Abbott TA, et al. Prediction of fracture risk in postmenopausal white women with peripheral bone densitometry: evidence from the National Osteoporosis Risk Assessment. J Bone Miner Res. 2002;17(12):2222-30.

6. Garnero P, Hausherr E, Chapuy MC, Marcelli C, Grandjean H, Muller C, et al. Markers of bone resorption predict hip fracture in elderly women: the EPIDOS prospective study. J Bone Miner Res. 1996;11(10):1531-8.

7. Garnero P, Sornay-Rendu E, Claustrat B, Delmas PD. Biochemical markers of bone turnover, endogenous hormones and the risk of fractures in postmenopausal women: the OFELY study. J Bone Miner Res. 2000;15(8):1526-36.

8. Gerdhem P, Ivaska KK, Alatalo SL, Halleen JM, Hellman J, Isaksson A, et al Biochemical markers of bone metabolism and prediction of fracture in elderly women. J Bone Miner Res. 2004;19(3):386-93.

9. van Daele PL, Seibel MJ, Burger H, Hofman A, Grobbee DE, van Leeuwen JP, et al. Case-control analysis of bone resorption markers, disability, and hip fracture risk: the Rotterdam study. Bmj. 1996;312(7029):482-3.

10. Rosen CJ, Hochberg MC, Bonnick SL, McClung M, Miller P, Broy S, et al. Treatment with once-weekly alendronate $70 \mathrm{mg}$ compared with onceweekly risedronate $35 \mathrm{mg}$ in women with postmenopausal osteoporosis: a randomized double-blind study. J Bone Miner Res. 2005;20(1):141-51.
11. Adami S, Giannini S, Giorgino R, Isaia G, Maggi S, Sinigaglia L, et al. The effect of age, weight, and lifestyle factors on calcaneal quantitative ultrasound: the ESOPO study. Osteoporos Int. 2003;14(3):198-207.

12. Blanchet $C$, Giguere $Y$, Prud'homme D, Turcot-Lemay L, Dumont M, Leduc $\mathrm{G}$, et al. Leisure physical activity is associated with quantitative ultrasound measurements independently of bone mineral density in postmenopausal women. Calcif Tissue Int. 2003;73(4):339-49.

13. Devine A, Dhaliwal SS, Dick IM, Bollerslev J, Prince RL. Physical activity and calcium consumption are important determinants of lower limb bone mass in older women. J Bone Miner Res. 2004;19(10):1634-9.

14. Fukuharu M, Sato J, Ohsawa I, Oshida Y, Kuriki K, Shibata K, et al. Effects of lifestyle factors on ultrasonographically determined bone health in Japanese women. Public Health. 2001;115(2):146-51.

15. Landin-Wilhelmsen K, Johansson S, Rosengren A, Dotevall A, Lappas G, Bengtsson BA, et al. Calcaneal ultrasound measurements are determined by age and physical activity. Studies in two Swedish random population samples. J Intern Med. 2000;247(2):269-78.

16. Lin JD, Chen JF, Chang HY, Ho C. Evaluation of bone mineral density by quantitative ultrasound of bone in 16,862 subjects during routine health examination. Br J Radiol. 2001:74(883):602-6.

17. Iwamoto J, Takeda T, Ichimura S. Relationships among physical activity, metacarpal bone mass, and bone resorption marker in 70 healthy adult males. J Orthop Sci. 2002;7(1):6-11.

18. Welsh $\mathrm{L}$, Rutherford OM. Hip bone mineral density is improved by highimpact aerobic exercise in postmenopausal women and men over 50 years. Eur J Appl Physiol Occup Physiol. 1996:74(6):511-7.

19. Woitge HW, Scheidt-Nave C, Kissling C, Leidig-Bruckner G, Meyer K, Grauer $A$, et al. Seasonal variation of biochemical indexes of bone turnover: results of a population-based study. J Clin Endocrinol Metab. 1998;83(1):68-75.

20. Yamazaki S, Ichimura S, Iwamoto J, Takeda T, Toyama Y. Effect of walking exercise on bone metabolism in postmenopausal women with osteopenia/ osteoporosis. J Bone Miner Metab. 2004;22(5):500-8.

21. Kitagawa J, Nakahara Y. Associations of daily walking steps with calcaneal ultrasound parameters and a bone resorption marker in elderly Japanese women. J Physiol Anthropol. 2008;27(6):295-300.

22. Chen JS, Cameron ID, Cumming RG, Lord SR, March LM, Sambrook PN, et al. Effect of age-related chronic immobility on markers of bone turnover. J Bone Miner Res. 2006;21(2):324-31.

23. Huang C, Ross PD, Wasnich RD. Vertebral fracture and other predictors of physical impairment and health care utilization. Arch Intern Med. 1996;156(21):2469-75

24. Camargos MC, Machado CJ, Rodrigues RN. Life expectancy among elderly Brazilians in 2003 according to different levels of functional disability. Cad Saude Publica. 2008;24(4):845-52.

25. Santos-Eggimann B, Cuenoud P, Spagnoli J, Junod J. Prevalence of frailty in middle-aged and older community-dwelling Europeans living in 10 countries. J Gerontol A Biol Sci Med Sci. 2009;64(6):675-81.

26. Ahacic K, Parker MG, Thorslund M. Mobility limitations in the Swedish population from 1968 to 1992: age, gender and social class differences. Aging (Milano). 2000;12(3):190-8

27. Sun W, Watanabe M, Tanimoto Y, Kono R, Saito M, Hirota C, et al. Assessment of the best gait parameter in relation to bone status in community-dwelling young-old and old-old women in Japan. Arch Gerontol Geriatr. 2009;49(1):158-61.

28. Sorva A, Valimaki M, Risteli J, Risteli L, Elfving S, Takkunen H, et al. Serum ionized calcium, intact PTH and novel markers of bone turnover in bedridden elderly patients. Eur J Clin Invest. 1994;24(12):806-12.

29. Bischoff $H$, Stahelin HB, Vogt $P$, Friderich $P$, Vonthein $R$, Tyndall A, et al. Immobility as a major cause of bone remodeling in residents of a long-stay geriatric ward. Calcif Tissue Int. 1999;64(6):485-9. 